\section{Grundzüge der Umsetzung des EFQM in der Suchtrehabilitation am Beispiel des Qualitätsmanagements im Verein Therapiehilfe e. V.}

\begin{abstract}
Zusammenfassung: Seit 1994 nutzt der in der Suchtrehabilitation in Hamburg und Schleswig-Holstein arbeitende Träger Therapiehilfe e.V. das Qualitätsmanagement-Modell der European Foundation for Quality Management (EFQM), Brüssel, zur kontinuierlichen Qualitätsentwicklung in all seinen Einrichtungen. Der Artikel gibt einen Überblick über die wesentlichen Gründe zur Einführung und stellt einige praktische Beispiele vor.
\end{abstract}

Schlüsselwörter: EFQM - Suchtrehabilitation - Qualitätsmanagement

Essential Features of the Transmission into Action of the European Foundation for Quality Management (EFQM, Brussels) in Addiction Rehabilitation at the Example of the Quality Management in the Organization Therapiehilfe e. V.: Working in the field of addiction rehabilitation in Hamburg and Schleswig-Holstein the quality management model of the EFQM, Brussels, is used for facilities for the continuous quality development into all his treatment centers. The article provides main aspects about the essential reasons for the introduction of EFQM to Therapiehilfe e. V. and demonstrates some concrete examples.

Key words: EFQM - Addiction Rehabilitation - Quality Management

Zu Beginn einer ernsthaften Diskussion über die Einführung von Qualitätssicherungsmaßnahmen (QS) im Jahr 1994 hat sich der Verein Therapiehilfe gefragt: „Was kann man von so einem Prozess haben oder wird das etwa nur gemacht, weil es verlangt wird?“ Und: Wenn wir QS einführen, „machen wir gerade das, was man braucht“, in einer Art Krisenbewältigungsstrategie, oder lassen sich nicht vielmehr gerade Qualitätssicherung und eigene Interessen zur Bewältigung der Zukunftsaufgaben sinnvoll verbinden?

Im Jahr 1989 ist die Qualitätssicherung in der stationären Versorgung in $§ 137$ SGB V gesetzlich verankert worden. Für den zweiten großen Leistungsbereich der Sozialhilfeleistun-

Suchttherapie 2000; $1: 173-175$

(c) Georg Thieme Verlag Stuttgart · New York

ISSN 1439-9903
Dieter Adamski, Karin Harries-Hedder

Therapiehilfe e.V., Hamburg gen fand dies eine Entsprechung in der Novelle des §93 BSHG und für die Jugendhilfe im „neuen“ KJHG.

Das „5-Punkte-Programm der Rentenversicherungsträger“, die Debatte um Leistungsverträge und die notwendigen „Leistungsvereinbarungen zum §93 BSHG“, die allerorts geführt worden sind, waren Ausdruck dieser Entwicklung.

Es war nicht einfach, sich hinzusetzen und diese Entwicklung im Verein voranzutreiben. Viele MitarbeiterInnen haben auch heute noch, vier Jahre nach dem Beginn der Arbeit mit dem EFQM-Modell ${ }^{1}$, eine kritische Distanz zu den Maßnahmen und Begrifflichkeiten, die einen Verein mit gesellschaftlichen Aufgaben in die Nähe einer Marktorientierung bringen. Diese erscheint ihnen für ihre Arbeit teilweise unangemessen und Menschenbilder sowie die gewachsene Professionalität eigener Programmatik scheinen sich dort nicht wiederzufinden.

Dass Qualität ausgerechnet in Zeiten erhöhten Spardrucks gesichert werden soll, um weitere Einsparpotenziale auszuloten, macht den ganzen Prozess immer wieder schwierig.

Für unsere Entscheidung für EFQM waren folgende Vorteile ausschlaggebend:

- Es trägt zur ständigen, dynamischen Weiterentwicklung des Vereins bei.

- Es fördert die Entwicklung gemeinsamer Wertestandards und verbindet alle Maßnahmen in einer gemeinsamen Qualitätsentwicklung aller unserer Einrichtungen. Es verhilft zur Synchronisation der Entwicklungen im Verein.

- Es ist integrationsfähig. Es können sowohl Anforderungen externer Kunden (z. B. das 5-Punkte-Programm der Rentenversicherungsträger, eine notwendige ISO-Zertifizierung oder auch veränderte Bedarfe der Patienten) im Rahmen des Modells qualifiziert umgesetzt werden.

\footnotetext{
${ }^{1}$ EFQM-Modell der European Foundation for Quality Management, Brüssel. Das EFQM ist ein Instrument zur strukturierten und systematischen Selbstbewertung des Qualitätsmanagements von Einrichtungen des Gesundheitswesens. Das EFQM ist ein mehrdimensionaler, dynamischer Ansatz, der sowohl Organisations- als auch Leistungsdimensionen enthält und so zur Abbildung, Bemessung und zum Vergleichen aller Ebenen einer Organisation nützlich ist. Nach dem Grundsatz „Messen ist Wissen“ soll Qualitätsentwicklung in 9 Dimensionen im Rahmen von Selbstevaluationsverfahren ermöglicht werden. „Messen ist Wissen“ heißt hier aber nicht, wie viel wovon, sondern findet sich in Bewertungsmaßstäben von Vorgehen - Ergebnissen - und Umfang der Tätigkeit wieder.
} 
- Es ist flexibel in der Handhabung, d.h. es gibt Möglichkeiten, das Modell situationsangemessen und den eigenen Rahmenbedingungen entsprechend umzusetzen.

Therapiehilfe e.V. ist ein Suchthilfeträger, der in Hamburg und den angrenzenden Landkreisen Schleswig-Holsteins vorwiegend Einrichtungen der Drogenhilfe, mit einer Vielzahl unterschiedlicher Leistungssegmente, betreibt. Die vergangenen Jahre sind durch ein stetiges Wachstum geprägt worden.

Wie man der Tabelle entnehmen kann, kommen zur Zeit rund $60 \%$ der Einnahmen durch das Erbringen von Gesundheitsdienstleistungen im Rehabilitationsbereich zustande. Nur $40 \%$ werden im Rahmen der Grundversorgung, also Streetwork, aufsuchende Sozialarbeit, Beratung und Nachbetreuung über noch nicht einzelfallbezogene Preise, eingeworben.

Damit wird Folgendes betrieben:

- ein Krankenhaus

- eine Fachklinik

- Rehabilitationseinrichtungen nach §111 SGB V

- Einrichtungen im Rahmen des §39 BSHG

- Einrichtungen im Rahmen der Jugend- und Erziehungshilfe nach dem KJHG

- und die oben erwähnten Zuwendungsprojekte

Dies alles wird in zwei Bundesländern realisiert, und oft mit mehreren Programmen bzw. auch Leistungssegmenten innerhalb einer Einrichtung. In der Konsequenz heißt das, dass in den Einrichtungen und Programmen sehr unterschiedliche Anforderungen an die Qualität unserer Dienstleistungen von außen formuliert werden. Diese verschiedenartigen Anforderungen von Behörden, Rentenversicherungsträgern und Krankenkassen sind nicht aufeinander abgestimmt und verursachen somit einen großen zusätzlichen Arbeitsaufwand in allen Einrichtungen.

Wenn Qualitätssicherung in einer solchen Organisation betrieben wird, muss das Verbindende herausgearbeitet werden, um zu einem gemeinsamen Verständnis von QS zu kommen. Unserer Auffassung nach gibt es in jeder Organisation eine immanente Kultur der Qualität. Bei uns wird sie durch Werthaltungen, die in einem Menschenbild der humanistischen Psychologie gebunden sind, psychotherapeutisches Methodenwissen, Selbstreflexion der Arbeit durch Supervision, Beziehung als tragendes Element der Behandlungs- und Betreuungsprozesse, zeitliche Kontinuität im Kontakt und dem Bemühen um Veränderung von Lebenssituationen, ausgedrückt.

Tab. 1

\begin{tabular}{llll}
\hline 1990 & 6 Einrichtungen & $\begin{array}{l}\text { ca. } 54 \\
\text { Mitarbeiterlnnen }\end{array}$ & $\begin{array}{l}\text { davon ca. } \\
70 \% \text { Zuwendungen } \\
\text { davon ca. }\end{array}$ \\
1992 & 10 Einrichtungen & $\begin{array}{l}\text { ca. } 125 \\
\text { Mitarbeiterlnnen }\end{array}$ & $\begin{array}{l}55 \% \text { Zuwendungen } \\
\text { davon ca. }\end{array}$ \\
1996 & 21 Einrichtungen & $\begin{array}{l}\text { ca. } 190 \\
\text { Mitarbeiterlnnen }\end{array}$ & $\begin{array}{l}30 \% \text { Zuwendungen } \\
\text { davon ca. }\end{array}$ \\
2000 & \multirow{2}{*}{30 Einrichtungen } & $\begin{array}{l}\text { ca. } 240 \\
\text { Mitarbeiterlnnen }\end{array}$ & $40 \%$ Zuwendungen
\end{tabular}

Dass dies nun dokumentiert, gemessen, belegt und mit anderen verglichen werden soll, war vielen MitarbeiterInnen am Anfang dieser Prozesse unverständlich.

Auf diesem Hintergrund begreifen wir unsere Aufgabe als Management, Lösungsversuche $\mathrm{zu}$ erarbeiten und $\mathrm{zu}$ initiieren, die in der Lage sind, den Rahmen zur Vermittlung solcher Wertkonflikte zwischen innen und außen darzustellen. Organisationsentwicklung und Qualitätsentwicklung sind in unserer Sichtweise eng verknüpft und nur machbar, wenn es gelingt, Partizipation am Prozess für die Kollegen und Kolleginnen zu ermöglichen.

QS wird in diesem Bereich derzeit nur funktionieren, wenn sich Vorstände und die jeweils oberste Leitungsebene entschließen, QS zu initiieren.

Konkret heißt das bei Therapiehilfe, dass das Thema Qualitätssicherung grundsätzlich als klare Leitungsaufgabe definiert und versucht wird, die Qualitätssicherung prozessorientiert zu initiieren. Wir wollen damit, durch ein prozessstrukturierendes Ineinandergreifen von Top-Down- und Bottom-Up-Ansatz, in einen andauernden Prozess der Qualitätsentwicklung einsteigen.

Qualitätszirkel scheinen hier, genauso wie verschiedene Projektarbeitsgruppen, geeignete Instrumente zur Verwirklichung gerade auch der Bottom-Up-Strategie zu sein.

\section{Entängstigung der Mitarbeiterinnen über den Umgang mit Ergebnissen}

Im Vorfeld kamen immer wieder die Fragen: Was geschieht mit den Ergebnissen? Missbraucht die Geschäftsleitung sie?

Der modellhafte Umgang war und ist also zu Anfang der Einführung eines solchen Systems von großer Bedeutung. Um als Beispiel zu dienen, hat die Leitungsrunde des Vereins als erster Bereich eine Selbstbewertung durchgeführt. So fungiert die Leitung als Modell. Die Ergebnisse und daraus resultierende Pläne sind allen MitarbeiterInnen zugänglich. Ziel ist eine positive Weiterentwicklung, ein Wachstum der Einrichtungen und kein Bekritteln von Bewertungen. Folgender Umgang mit den Bewertungen soll im gesamten Verein erreicht und modellhaft vorgelebt werden: Ergebnisse der Selbstbewertungsprozesse sollen diskutiert werden und nicht in Schubladen oder Werbebroschüren verschwinden. Zentrale Fragestellungen sind: Warum ist dieser oder jener Wert in der Selbstbewertung erreicht worden? Hat eine der EFQM-Dimensionen de facto wenig Bedeutung? Wurde etwas vernachlässigt? Hat es Sinn, hier etwas zu verändern, und wie könnte das konkret geschehen?

\section{Zur Umsetzung}

Aus unserer Sicht mussten bei der Umsetzung mehrere Faktoren berücksichtigt werden:

- Eine umfassende Vorbereitungs- und Entscheidungsphase für das neue System im gesamten Träger, da die Umsetzung nur unter aktiver Beteiligung vieler MitarbeiterInnen und aller hierarchischer Ebenen stattfinden konnte.

- Es musste relativ schnell und breitflächig eingeführt werden, da die Vorteile von EFQM nur durch die Anwendung 
für die Einrichtung erlebbar und damit motivierend wirken.

- Es musste durch die Erfahrung des Umgangs mit der Selbstbewertung Entängstigung stattfinden. Da auch durchaus geringe Bewertungen (niedrige Werte) in einigen Dimensionen normal sind, muss gerade hier, besonders bei den ersten Ergebnissen, mit viel Sensibilität von Seiten der Leitungskräfte und der Geschäftsführung vorgegangen werden. Es musste, parallel zur Selbstbewertung, zunächst auch der Umgang mit den Dimensionen und das neue Vokabular erarbeitet und - wo möglich - entsprechend angewandt werden.

\section{Das EFQM-Modell wurde parallel eingeführt:}

1. Ausbildungen und Fortbildungen: Alle vorhandenen Ressourcen wie der „Therapiehilfe-Tag“, ein jährlich stattfindendes, internes Seminar für sämtliche MitarbeiterInnen und das schon existierende „Fortbildungsprojekt der Therapiehilfe“ wurden über Jahre genutzt, um die MitarbeiterInnen mit dem EFQM vertraut zu machen.

2. Die Geschäftsführung und 16 MitarbeiterInnen wurden zu Assessoren des EFQM ausgebildet. Die Funktion der Qualitätsbeauftragten wurde als Stabsstelle der Geschäftsleitung geschaffen.

3. Ein „Qualitätsforum“ mit den 16 ausgebildeten EFQMAssessoren, unter Leitung der Qualitätsbeauftragten, wurde gegründet. Diese sind in ihren Einrichtungen für die Umsetzung der Qualitätsprojekte zuständig. Zusätzlich moderieren sie im Gesamtverein in wechselnder Besetzung die Selbstbewertungsprozesse.

4. Vorhandene Strukturen - wie regelmäßig wiederkehrende Besprechungstermine und Konferenzen - wurden dazu genutzt, unter einem zusätzlichen Tagesordnungspunkt über die Qualitätsentwicklung zu informieren, darüber zu diskutieren und eine Abstimmung über Qualitätsprojekte zu erzielen.

\section{Qualitätsforum}

Seit Anfang 2000 hat das Qualitätsforum unter Leitung der Qualitätsbeauftragten angefangen zu arbeiten. Erstes Ziel des Forums ist die Umsetzung der Selbstbewertung in allen Einrichtungen. In das Forum wurden nicht nur Leitungskräfte, sondern auch Teammitglieder ohne Leitungsfunktion aus den Einrichtungen zur Teilnahme gebeten. Dies hat einerseits mit geringen personellen Ressourcen $\mathrm{zu}$ tun, andererseits bietet es die Chance für engagierte MitarbeiterInnen, strukturierend an der Entwicklung des Vereins teilzunehmen. Dies ist nicht nur eine neue spannende Arbeit, sondern auch eine zusätzliche Qualifikation für die MitarbeiterInnen. Zudem wirken Teammitglieder, die sich für das EFQM begeistern lassen, oft motivierender auf die KollegInnen als Leitungskräfte.

Die Qualitätsbeauftragte und die ForumsmitarbeiterInnen haben einen Zeitplan für die Selbstbewertung aller Einrichtungen erstellt. Die Selbstbewertung erfolgt also nicht mit Hilfe von externen Fachleuten, wie meist üblich, sondern je 2 Mitglieder des Forums moderieren die Selbstbewertung und erstellen den Bericht. Die Auswertung und die daraus resultierende Planung für die jeweilige Einrichtung werden dann im Einrichtungsteam erarbeitet.

\section{Zielvereinbarungen}

Anfang des Jahres werden mit allen EinrichtungsleiterInnen Zielvereinbarungen für das kommende Jahr erarbeitet. Seit 1999 wird das Inventar des EFQM zur Strukturierung der Zielvereinbarungen anhand der vorgegebenen Dimensionen genutzt. Es wurde ein Leitfaden entwickelt, der nach den EFQM-Dimensionen gegliedert ist, anhand dessen werden die anliegenden Themen bearbeitet und danach wird eine Vereinbarung geschlossen.

Unsere Erfahrung ist, dass ein sehr zügiges und gut strukturiertes Arbeiten anhand der Dimensionen möglich ist und alle Arbeitsfelder in dieser Struktur erfasst werden.

\section{Jahresberichte}

Ein weiteres Beispiel sind die Jahresberichte der Einrichtungen, die inzwischen zunehmend nach den EFQM-Dimensionen strukturiert werden. Die Jahresberichte werden von den Teams der Einrichtungen erstellt. Auch hier haben KollegInnen das neue Handwerkzeug des EFQM genutzt.

Wir stehen bei der Umsetzung des EFQM für den Träger erst am Anfang und sehen etliche Unzulänglichkeiten. Trotzdem scheinen die bislang gesammelten Erfahrungen unsere These $\mathrm{zu}$ bestätigen, dass man einfach mit der Umsetzung auf verschiedenen Ebenen beginnen kann - und muss. Wir haben uns bemüht, die Systematik des EFQM möglichst in den bestehenden Alltag zu integrieren.

Wir folgen seit Einführung des EFQM nicht nur den geforderten Rahmenbedingungen, sondern nutzen das Instrument für die Fortentwicklung einer Unternehmenskultur.

Der Umgang mit dem neuen Instrument kann nur durch Erfahrungen gelebt und gelernt werden. Nur wenn möglichst viele MitarbeiterInnen einen Vorteil durch das neue System erfahren, wird es sich in der Organisation etablieren. Wir sind gespannt und noch optimistisch und hoffen, mit diesem Managementsystem die Anforderungen der Zukunft als Gesamtverein gut zum Nutzen der KlientInnen umsetzen zu können.

\section{Dieter Adamski}

\section{Karin Harries-Hedder}

Therapiehilfe e. V.

Hasselbrookstraße 4a

22089 Hamburg 